\title{
Tranches de vie en Amérique latine
}

\section{Jean Martin}

Dr méd., membre de la rédaction

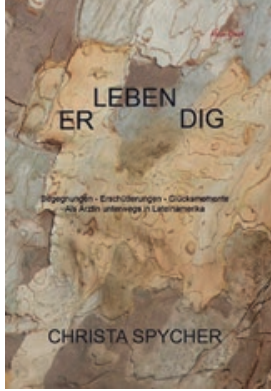

Christa Spycher ER-LEBEN-DIG Als Ärztin unterwegs in Lateinamerika

Mein Buch, 2020 147 pages
Christa Spycher, fille d'un médecin de famille de Berthoud, a fait avec son mari Peter l'essentiel de sa carrière dans le domaine du développement, avec la coopération suisse. Ils ont vécu près de 20 ans en Amérique latine. Après six ans dans la capitale péruvienne Lima, ils ont été trois ans dans un projet agro-forestier sur la rive de l'Ucayali, affluent de l'Amazone, et plus tard sept ans au Honduras et au Nicaragua - avec deux périodes intermédiaires à la centrale de Berne. Retour au pays pour de bon en 1998; alors que son mari y concluait sa carrière, la Dre Spycher s'est beaucoup engagée dans le domaine du planning familial - reprenant une activité liée aux problématiques de la femme dont elle s'était intensément préoccupée en Amérique centrale. Officiellement épouse de coopérant, mère de trois filles, l'auteure a aussi été une professionnelle apportant, dans des conditions difficiles, ses compétences médicales et son dynamisme pour soigner et participer à des actions de formation. Elle le raconte avec talent, ne traînant jamais en longueur, passant avec légèreté sur les mauvais moments.

Personnellement, je suis touché par cet ouvrage pour avoir également travaillé quelques années en Amazonie péruvienne, dans un hôpital près de Pucallpa. Mais aussi parce que j'ai rencontré, vers 2000, Christa Spycher dans le cadre de "Santé Sexuelle Suisse».

ER-LEBEN-DIG est un récit s'étendant sur trois décennies vécues au contact de pays et populations hôtes de

\section{Christa Spycher relate toujours avec simplicité et empathie, sans cacher les difficultés et les occasionnelles déceptions.}

projets de développement. En milieu rural et urbain, dans des conditions matérielles et sous des régimes politiques divers. Les expériences professionnelles, personnelles, familiales de l'auteure sont contées de manière proche des gens et de leurs manières et lieux de vie. Elle relate toujours avec simplicité et empathie, sans cacher les difficultés et les occasionnelles déceptions. Il ne s'agit pas de souvenirs de type «anciens combattants», mais de vécu constructif, ouvert, qui donne à réfléchir au sens de notre vie et de nos actions. Grandes dimensions de ce parcours: trois ans en Amazonie (pour accéder à leur site, une douzaine d'heures de canot à moteur sont nécessaires depuis l'amont, le double depuis l'aval); les apprentissages socio-culturels; la confrontation à la précarité, voire la misère, omniprésente et à ses multiples conséquences; le travail avec les femmes dans plusieurs pays, y compris au Nicaragua où la révolution sandiniste (avant qu'elle ne

Bien que confrontée à la misère, l'autrice n'offre pas de récit sombre, au contraire. C'est plein de vie, de chaleur humaine.

perde son âme) a eu de remarquables succès dans l'alphabétisation, la promotion communautaire, la lutte contre la violence domestique.

Dans une recension, on a envie de parler d'épisodes exemplaires, et il y en a beaucoup. Je n'en cite qu'un qui m’a touché: «Mili est une femme de 32 ans qui a un handicap à la marche, avec 9 enfants. Je lui rends visite, son dernier est malade avec une forte fièvre. Comment aider, par quoi commencer? Je demande où est Rosita, l'aînée, 13 ans. Mili se tortille... Rosita vit maintenant avec un jeune policier, trois rues plus loin... Sur le moment, je ne veux pas comprendre. Mais j'ai la situation sous les yeux... Rosita a été vendue. Cela me taraude, je sens du désespoir [...] Des années plus tard, en Suisse, confrontée à la violence faite aux enfants et aux femmes dont nous nous occupions en équipes interdisciplinaires, il m'est revenu encore et encore combien, dans les circonstances des barrios (quartiers pauvres), j'avais insuffisamment saisi la réalité de la violence, combien je m'étais instinctivement protégée de la voir» (pp. 98-99). Même avec une expérience longue et solide, on reste parfois inhibé, incrédule, parce que les faits choquent trop quand on doit les admettre.

Mais l'essentiel de ce que raconte Christa Spycher n'est pas sombre, au contraire. C'est plein de vie, de chaleur humaine, de découverte de l'autre, d'autres cultures et réactions devant les péripéties de l'existence. Des choses se construisent, progressent - avec des hauts et des bas.

A mon sens, chaque fois qu'une personne prend la plume pour transcrire une existence bien remplie, il faut s'en féliciter. De plus, sans doute, ce récit peut être de grande aide et d'intérêt pour celles et ceux qui aujourd'hui s'engagent dans des activités de coopération. 